\title{
Should I stay or should I go? Maximizing Lifetime with Relays
}

\author{
Peter Terlecky, Brian Phelan, Amotz Bar-Noy, Theodore Brown, Dror Rawitz
}

\begin{abstract}
We consider the problem of maximizing the lifetime of a wireless connection between a transmitter and a receiver using mobile relays. Initially, all relays are positioned arbitrarily on the line between the transmitter and the receiver and have arbitrary battery capacities. Energy is consumed in proportion to the distance traveled for mobility and in proportion to an exponential function of the distance over which information is sent for communication. The objective is to find positions and thus transmission ranges for the nodes that maximize the lifetime of the network. We study two models, the single deployment model and the multiple deployment model. We show how to compute an optimal solution for the case of no movement cost for both models. When there is a cost for movement we develop some structure for multiple deployment model. We consider a discrete version of the single deployment model, in which relays are deployed on grid points. We provide two algorithms for this case: a dynamic programming algorithm and a binary search algorithm. We prove that both algorithms are FPTASs for the non-discrete problem, if batteries are not too small. We develop a number of heuristics for the multiple deployment model. Our simulations demonstrate the benefit of moving over remaining at initial locations.
\end{abstract}

Index Terms—Deployment; Mobility; Network Lifetime; Relays; Sensor Networks.

\section{INTRODUCTION}

Keeping the network alive for as long as possible is one of the main goals in any Wireless Sensor Network (WSN). This paper uses recent advances in node mobility (see El-Moukaddem et al. [6] and the references therein) to maximize the lifetime of a WSN using mobile-relay-based algorithms that move mobile relays to optimal locations along the line between a transmitter and a receiver.

Several papers address the problem of optimal placement of relays between a source and a destination to optimize other objective functions. Appuswamy et al. [1] optimize the capacity of the implied logical channel between the source and the destination under some interference model. This paper allows only grid placements of relays with the same, non-adjustable transmission range and does not consider movement cost.

Goldenberg et al. [7] prove that, for equal battery levels, the optimal locations for the relays on the line are equidistant apart and present an algorithm for moving nodes to their optimal locations using information from 1-hop neighbors only. They prove that

- P. Terlecky is with the Mathematics Department, The Graduate Center, City University of New York, NY, 10016. E-mail: pterlecky@gc.cuny.edu

- B. Phelan, A. Bar-Noy, and T. Brown are with the Computer Science Department, The Graduate Center, City University of New York, NY, 10016

E-mail: \{bphelan,tbrown\}@gc.cuny.edu, amotz@sci.brooklyn.cuny.edu

- D. Rawitz is with the School of Electrical Engineering, Tel-Aviv, Isreal, 69978

E-mail: rawitz@eng.tau.ac.il their algorithm preserves the connections between all the relays.

Jiang et al. [9] develop a number of algorithms to speed up the rate of convergence and, hence, minimize the number of iterations required to move each of the nodes to their optimal locations. We extend the above works by taking into consideration the remaining battery life in each node and the effect that different mobility costs will have on the optimal locations of the nodes.

El-Moukaddem et al. [6] consider using mobile relays to enhance the lifetimes of existing network routes between static nodes in a $2 \mathrm{D}$ plane. Each relay can assist a single link between two nodes. They include realistic costs for movement and transmission and take into consideration the battery power remaining in each of the nodes.

In this paper we study the problem of maximizing the lifetime of a wireless connection between a stationary transmitter and a stationary receiver using mobile relays that are initially positioned arbitrarily on the line between the transmitter and the receiver and have arbitrary battery levels. For example, the relays may be initially located at the transmitter that deploys them to assist it with a transmission. We consider several variants of the problem depending on the deployment method. In many applications, [7], [9], [17], relays are allowed to move only at time zero in order to decrease the amount of communication overhead between relays. It may also be the case that relays are initially deployed using an external agent, and cannot move by themselves. We refer to this model as the single deployment model. In the multiple deployment setting relays can move at any point in 
time.

Our contribution. We consider two network models, the first model corresponds to the case where relays are allowed to be set once, while the second model corresponds to the case where relays can be adjusted multiple times. In the first model relays can be deployed only at time 0 in an order preserving manner. The lifetime of the system is determined by the weakest link, namely by the lifetime of the relay whose battery is depleted first. This notion of Lifetime of First Death was consider by El-Moukaddem et al. [6]. We represent this problem as a nonlinear program. The second model allows multiple deployments. Relays readjust their transmission ranges after a deployment. In this case we wish to maximize the length of time the transmitter can communicate with the receiver, that is the Transmission Lifetime.

We show how to compute relay locations for both models when there is no cost for movement. It turns out that for this case there exists a solution which is optimal with respect to both models, namely there exists an optimal single deployment order preserving strategy that maximizes both notions of lifetime.

For non-zero movement cost, we develop some structure for the optimal solution. In particular, we show that there is no justification for movement unless a relay becomes dies and that in any optimal solution the transmitter must be the last node to die.

We consider a discrete version of the single deployment, in which relays must be deployed on grid points. Given any $\varepsilon>0$, we show that if batteries are not too small, there exists a grid density for which the discrete optimal lifetime of first death is within a factor of $(1+\varepsilon)$ from the optimal lifetime of first death. We provide two algorithms for the discrete problem. The first algorithm is a dynamic programming algorithm that computes an optimal solution, while the second conducts a binary search for the optimum. For the case where the batteries are not too small, both algorithms are FPTASs for the non-discrete problem, since their running times are polynomial in the size of the input and in $1 / \varepsilon$. We experimentally test whether multiple deployments can assist in maximizing transmission lifetime. We find that just a single deployment is necessary in maximizing transmission lifetime. We develop heuristics for maximizing transmission lifetime based on this result and based on our theoretical findings and show that they are well-performing in simulation. Finally, we consider the natural case where relays with different battery levels are to be deployed from the location of the transmitter (base station). We experimentally find that in a maximal lifetime of first death solution relays should be deployed so that relays with larger batteries travel farther.

Related work. The numerous uses of mobility in WSNs are discussed in Francesco et al. [5] along

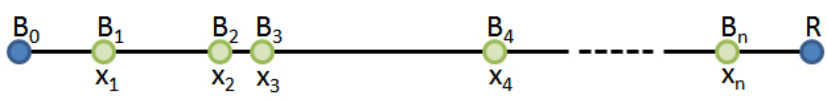

Fig. 1: $n$ relays on the line between the transmitter and the receiver.

with the challenges that arise when mobile nodes are introduced to a network such as maintaining connectivity. Along with mobile relays, other uses of mobility include mobile sinks and data MULEs.

Controlled sink mobility is considered by Basagni et al. [2], while predictable sink mobility is considered by Chakrabarti et al. [3], Chandra et al. [4] and Mhatre et al. [13]. Another approach considered in the literature is allowing the sink to move randomly as in Juang et al. [10] and Kim et al. [12].

Data MULEs were introduced by Shah et al. [15] and are further explored by Jain et al. [8].

Song et al. [16] build a prototype mobile node to demonstrate that mobile nodes are feasible. Kansal et al. [11] proposes using controlled mobility to optimize the power usage of a WSN and discusses a number of potential network features, for example tracks, that would make adding controlled mobility cost effective.

Paper organization. Formal descriptions of the problem, deployment methods, and network lifetimes are in given in Section 2. The case where movement is free of cost is analyzed in Section 3. Section 4 gives some structural results. Section 5 considers the discrete version of the single deployment model. We present some heuristics for maximizing transmission lifetime in Section 6. Our simulations are given in Section 7. Finally, Section 8 discusses areas for future research.

\section{Model and Preliminaries}

In this section we define the models and introduce the notation which will be used throughout the paper.

A transmitter would like to transmit information to a receiver. To aid in the transmission of information, $n$ mobile relays are distributed on the line of communication between the transmitter and the receiver (as in Fig. 1). Each relay has the task of passing along the information and thus maintaining the chain of communication. The distance between the transmitter and the receiver is denoted by $D$. We assume w.l.o.g. that the transmitter is located at 0 , while the receiver is located at $D$. The initial locations of the nodes are denoted by $x_{0}, x_{1}, \ldots, x_{n}$, where $x_{0}=0$ is the location of the transmitter.

The initial battery power of node $i \in\{0,1, \ldots, n\}$, is denoted by $B_{i}$. Communicating costs energy and the energy required depends entirely on the distance over which the information must be transmitted. Following the model of Moscibroda et al. [14], a node transmitting data over a distance $d$ invests $P(d)=d^{\alpha}$ energy 
per time unit, where $\alpha>1$ is a constant. The cost of mobility, or friction, is proportional to the distance traveled, $k$, as in Wang et al. [18], i.e. $M(k)=a k$, where $a$ is a constant.

We allow relays to occupy the same location. If two or more nodes are located at the same point, then one of them (say the node with the highest index) must transmit to the next live node which is not at the same location, while the other nodes do not consume any power.

Using the above definitions, we represent the network by the vector

$$
N=\left\langle D, B_{0}, \ldots, B_{n}, x_{1}, \ldots, x_{n}, \alpha, a\right\rangle,
$$

which gives the distance from transmitter to receiver, the initial battery powers and positions of the nodes, and the cost parameters, $\alpha$ and $a$. When $a=0$ we say that there is no friction.

\subsection{Single Deployment and Lifetime of First Death}

Our first model corresponds to the case where relays are allowed to be set once. Relays are to be deployed at time 0 after which transmission may commence. The lifetime of the network is determined by its weakest link, namely by the lifetime of the relay whose battery is depleted first, thus breaking the chain of communication.

We have $n$ relays initially located at $x_{1}, x_{2}, \ldots, x_{n}$ between 0 and $D$ on the line. The relays are to be deployed to some locations $y_{1}, y_{2}, \ldots, y_{n}$. Moving relay $i$ from location $x_{i}$ to location $y_{i}$ decreases relay $i$ 's battery by $a\left|x_{i}-y_{i}\right|$. Let $B_{i}^{\prime}$ be the new battery level of relay $i$ after the movement, namely $B_{i}^{\prime}=B_{i}-a\left|x_{i}-y_{i}\right|$. (Clearly $B_{0}^{\prime}=B_{0}$.) Let right ${ }_{i}$ denote the left-most node to the right of $i$, after the relays are deployed. A node $i \in\{0, \ldots, n\}$ must transmit to right ${ }_{i}$. Let $d_{i}$ be the transmission range of node $i$, for every $i \in\{0, \ldots, n\}$, namely $d_{i}=y_{\text {right }_{i}}-y_{i}$, for every $i \in\{0, \ldots, n\}$, where $y_{\text {right }_{i}}=D$ if the receiver is the rightmost node to the left of node $i$.

Once the relays arrive at locations $y=\left(y_{1}, y_{2}, \ldots, y_{n}\right)$, they may begin transmitting and must transmit from their respective locations for the duration of their lifetimes. The lifetime of relay $i$ is defined as $L_{i}\left(y_{i}\right)=B_{i}^{\prime} / d_{i}^{\alpha}$. The lifetime of the system given the deployment to $y$ is the time in which the first relay dies, and it is defined as

$$
L_{F}(N, y)=\min _{0 \leq i \leq n} L_{i}\left(y_{i}\right) .
$$

We shall refer to this lifetime as Lifetime of First Death. This notion of network lifetime was considered by El-Moukaddem et al. [6] for a max data mobile relay configuration.

The MAXIMUM LIFETIME OF FIRST DEATH problem (MAXFD) is the problem of finding a deployment that maximizes the lifetime of first death.
In this paper we focus on the no swapping case, where the relays are to be deployed to some locations $y_{1}, y_{2}, \ldots, y_{n}$ preserving the initial order between relays, i.e. $x_{i} \leq x_{j}$ implies $y_{i} \leq y_{j}$, for every $i \neq j$. In this case, $d_{i}=y_{i+1}-y_{i}$ for $i \in\{0, \ldots, n-1\}$ and $d_{n}=D-y_{n}$. This problem can be represented by the following nonlinear (and non-convex) program:

$$
\begin{aligned}
& \max _{y_{1}, \ldots, y_{n}} \min \left\{\frac{B_{0}}{y_{1}^{\alpha}}, \frac{B_{1}-a\left|x_{1}-y_{1}\right|}{\left(y_{2}-y_{1}\right)^{\alpha}}, \ldots, \frac{B_{n}-a\left|x_{n}-y_{n}\right|}{\left(D-y_{n}\right)^{\alpha}}\right\} \\
& \text { s.t. } 0 \leq y_{1} \leq \cdots \leq y_{n} \leq D
\end{aligned}
$$

The reason for focusing on this case is threefold. First, there are applications in which swapping is disallowed. For example, Kansal et al. [11] suggest using relays on a track. Second, in some scenarios adding this no swapping restriction gives additional structure that may be used for solving the problem without affecting the solution space. For instance, when $n$ identical relays are initially located at the same point. Finally, we show (in Section 3) that swapping is unnecessary in the non-friction case.

Another nice implication of the no-swapping assumption is multiple deployments do not help. More specifically, we can show that any solution where relays redeploy after time 0 can be replaced by a single deployment solution whose lifetime is not worse, see corollary 5.

Furthermore, we assume that all relays must participate in communication. This may have a drawback in that if any relay has a very small initial battery and a sizable transmission range, the lifetime of first death would be short. Note that if $B_{i} \geq a \max \left\{x_{i}, D-x_{i}\right\}$, for all $i$, then a relay may effectively deactivate by moving arbitrarily close to the next relay. Thus, deactivation is encompassed by the model given if $B_{i} \geq a \max \left\{x_{i}, D-x_{i}\right\}$, for all $i$. We also note that our FPTASs for MAXFD are based on a stronger assumption (see Section 5).

\subsection{Multiple Deployment and Transmission Life- time}

In our second model we assume relays can be deployed multiple times and can readjust their transmission ranges after a deployment or after the death of a relay. In this model, we wish to maximize the length of time the transmitter can communicate with the receiver. We call such a lifetime the Transmission Lifetime.

A solution, or solution path, is defined as $\mathcal{P}:=$ $\left\{x_{1}(t), \ldots, x_{n}(t)\right\}_{t=0}^{\infty}$, where $x_{i}(t)$ is the location of relay $i$ at time $t$ for $i \in\{1, \ldots, n\}$. Let $\operatorname{left}_{i}(t)$ and right $_{i}(t)$ denote the right-most node to the left of node $i$ and the left-most node to the right of $i$ at time $t$. A node $i \in\{0, \ldots, n\}$ must transmit to $\operatorname{right}_{i}(t)$. If relay $i$ dies at some time $t$ then the node $\operatorname{left}_{i}(t)$ increases its transmission range to transmit to right ${ }_{i}(t)$. The transmitting range of a live node $i$ at time $t$, denoted by 
$d_{i}(t)$, is the distance between that node and $\operatorname{right}_{i}(t)$, i.e., $d_{i}(t)=x_{\text {right }_{i}(t)}(t)-x_{i}(t)$. The remaining battery power of node $i$ at time $t$ is denoted by $B_{i}(t)$.

Let $X_{i}(t)$ be the total distance traveled by node $i$ up to time $t$ (note $X_{0}(t)=0 \forall t$ ). Using this notation, the lifetime of node $i$ under solution path $\mathcal{P}, L_{i}(\mathcal{P})$, satisfies:

$$
B_{i}=a X_{i}\left(L_{i}(\mathcal{P})\right)+\int_{0}^{L_{i}(\mathcal{P})} d_{i}(t)^{\alpha} d t
$$

A solution path $\mathcal{P}$ is feasible if (i) $x_{i}(0)=x_{i}$ for every $i \in\{1, \ldots, n\}$, and (ii) $x_{i}(t)=x_{i}\left(L_{i}(\mathcal{P})\right)$, for every $i \in\{1, \ldots, n\}$ and $t \geq L_{i}(\mathcal{P})$.

We define the Transmission Lifetime for a solution path $\mathcal{P}$ and network $N$, denoted by $L_{T}(N, \mathcal{P})$, to be the length of time the transmitter can send data to the receiver in a solution path $\mathcal{P}$ for a given network $N$. This is equivalent to the lifetime of the transmitter under a solution path $\mathcal{P}, L_{0}(\mathcal{P})$. Thus:

$$
L_{T}(N, \mathcal{P})=L_{0}(\mathcal{P}) .
$$

The Maximum TRANSMission Lifetime problem (abbreviated MAXTL) is the problem of finding a solution path $\mathcal{P}$ that maximizes the transmission lifetime of a given network $N$.

Observe that the maximum transmission lifetime is never smaller than the maximum lifetime of first death, namely

$$
\max _{y} L_{F}(N, y) \leq \max _{\mathcal{P}} L_{T}(N, \mathcal{P})
$$

for every network $N$, since $L_{F}(N, y) \leq L_{T}\left(N, \mathcal{P}_{y}\right)$, for every deployment $y$, where $\mathcal{P}_{y}$ is the solution path that corresponds to $y$, i.e., $\mathcal{P}_{y}$ satisfies $x_{i}(t)=y_{i}$ for every relay $i$.

\section{MaXTL AND MAXFD without Friction}

In this section we consider the no friction case, namely the case where $a=0$. Goldenberg et al. [7] consider the case where there is no friction and all nodes have equal battery power. They show that the energy cost function $P(d(t))$ is a non-decreasing convex function, and that the optimal positions of the relay nodes must lie entirely on the line between the source and the destination and must be evenly spaced along the line.

We can extend this result to the case of non-equal battery powers. We show that the lifetime of the network is optimized when we choose the transmission ranges $d_{i}(t)^{\prime} \mathrm{s}$ to be such that the lifetimes of all nodes are equal, i.e. $L_{i}=L_{j}, \forall i, j \in\{0, \ldots, n\}$ and fixed for the lifetime of the network: $d_{i}(t)=d_{i}$ for the lifetime (both transmission and first death) of the network. In this case, relays only need to move once to their optimal locations and transmission lifetime is equal to lifetime of first death.

Lemma 1. The transmission lifetime and lifetime of first death of a network with $n$ relays where there is no friction is maximized when $L_{i}=L_{j}$, for every $i, j \in\{0, \ldots, n\}$.
Proof: Let $T$ be the transmission lifetime of an optimal solution. Define $\overline{d_{i}}$ to be the time average of $d_{i}(t)$ from 0 to $T$ for each $i \in\{0, \ldots, n\}$ :

$$
\overline{d_{i}}=\frac{1}{T} \int_{0}^{T} d_{i}(t) d t .
$$

We first show that $\sum_{i=0}^{n} \overline{d_{i}}=D$ :

$$
\begin{aligned}
\sum_{i=0}^{n} \overline{d_{i}} & =\sum_{i=0}^{n} \frac{1}{T} \int_{0}^{T} d_{i}(t) d t \\
& =\frac{1}{T} \int_{0}^{T} \sum_{i=0}^{n} d_{i}(t) d t=\frac{1}{T} \int_{0}^{T} D d t=D .
\end{aligned}
$$

A feasible placement in which $i^{\prime}$ s range is $\overline{d_{i}}$ can be obtained by placing $i$ at $\sum_{j=0}^{i-1} \overline{d_{j}}$, for every $i$. We now show that for arbitrary $i$ :

$$
\int_{0}^{T}\left(\overline{d_{i}}\right)^{\alpha} d t \leq \int_{0}^{T} d_{i}(t)^{\alpha} d t
$$

This follows with the use of Jensen's Inequality:

$$
\begin{aligned}
\int_{0}^{T}\left(\overline{d_{i}}\right)^{\alpha} d t & =T \cdot\left(\overline{d_{i}}\right)^{\alpha} \leq T \cdot \overline{d_{i}(t)^{\alpha}} \\
& =T \cdot \frac{1}{T} \int_{0}^{T} d_{i}(t)^{\alpha} d t=\int_{0}^{T} d_{i}(t)^{\alpha} d t .
\end{aligned}
$$

It thus suffices to consider the solutions in which the transmission ranges $d_{i}(t), i \in\{0, \ldots, n\}$ are fixed for the duration of lifetime. Of all such solutions, the one in which all node lifetimes are equivalent, $L_{i}=L_{j}, \forall i, j \in\{0, \ldots, n\}$, maximizes the lifetime of the network.

Assume this is not the case. Consider the time $M$ when the transmitter dies. Note that if a relay dies before time $M$ the solution does not have fixed transmission ranges. Consider the leftmost relay which is still alive at time $M$. WLOG, assume it is relay $k$. Shift the first $k$ relays to the left by an amount $\delta>0$, where $\delta \leq \min \left\{d_{0}(M), \epsilon\right\}$ and $\epsilon=\sqrt[\alpha]{\frac{B_{k}}{M}}-d_{k}$, i.e., $\epsilon$ satisfies $B_{k} /\left(d_{k}+\epsilon\right)^{\alpha}=M$. The shift decreases $d_{0}$, while leaving $d_{1}, d_{2}, \ldots, d_{k-1}$ unchanged. Consequently the lifetime of this solution is greater than the lifetime of the optimal, a contradiction.

If $L_{i}=L_{j}, \forall i, j \in\{0, \ldots, n\}$, lifetime of first death equals transmission lifetime. Since lifetime of first death is never larger than transmission lifetime, it follows that lifetime of first death is maximized as well.

Theorem 2. If $a=0$, an optimal solution for MAXTL and MAXFD is obtained by placing relay $i$ at $\sum_{j=0}^{i-1} d_{j}$, where

$$
d_{i}=D \cdot \frac{\sqrt[\alpha]{B_{i}}}{\sum_{j=0}^{n} \sqrt[\alpha]{B_{j}}}
$$

for every $i \in\{1, \ldots, n\}$. The corresponding lifetime is $D^{-\alpha}\left(\sum_{j=0}^{n} \sqrt[\alpha]{B_{j}}\right)^{\alpha}$. 
Proof: Consider relay $i$. Due to Lemma 1 we know that $L_{i}=L_{j}$ for every $j \in\{0, \ldots, n\}$. It follows that $d_{j}=\sqrt[\alpha]{B_{j} / B_{i}} \cdot d_{i}$, for every $j \in\{0, \ldots, n\}$. Since $\sum_{j=0}^{n} d_{j}=D$, we have that

$$
\frac{d_{i}}{\sqrt[\alpha]{B_{i}}} \sum_{j=0}^{n} \sqrt[\alpha]{B_{j}}=D,
$$

which gives the result.

Corollary 3. If $a=0$, there exists a single deployment and no swapping optimal solution for MAXTL.

\section{MAXTL WITHOUT SWAPPING}

In this section, we consider MAXTL with friction. We provide some structure for this case by giving necessary conditions for any optimal solution.

First, we note that Theorem 2 does not hold in the non-zero friction case. Consider the case where we have one relay located at 0.25 with $B_{0}=B_{1}=1$ and $D=1$. Also, assume that $a=4$. This means that going from 0.25 to 0.5 depletes the relay. However, located at $[0.25,0.5)$ the relay dies before the transmitter.

We prove that in an optimal solution, relays do not need to move unless some relay dies.

Lemma 4. Given a MAXTL instance, any solution where a relay moves at a time that does not correspond to the death of another relay can be replaced by a stationary solution with at least as good a lifetime.

Proof: Assume that in an optimal solution no relay dies in the time interval $\left(t_{0}, t_{2}\right)$. Furthermore, assume that in this optimal solution, relays move at an instance $t_{1}$, where $t_{0}<t_{1}<t_{2}$ and are stationary otherwise.

Consider the time average location of relay $i$ from $t_{0}$ to $t_{2}$, which has moved from location $x_{i}\left(t_{0}\right)$ to $x_{i}\left(t_{1}\right)$ at time instance $t_{1}$ :

$$
\bar{x}_{i}=\frac{x_{i}\left(t_{0}\right) \cdot\left(t_{1}-t_{0}\right)+x_{i}\left(t_{1}\right) \cdot\left(t_{2}-t_{1}\right)}{t_{2}-t_{0}} .
$$

We claim that a solution that places relay $i$ at location $\bar{x}_{i}$ at time $t_{0}$ and does not move $i$ before $t_{2}$ is at least as good as the original solution. First, notice that $\bar{x}_{i} \leq \bar{x}_{i+1}$ for every $i$, namely relay ordering is maintained by the new solution. Also, since location $\bar{x}_{i}$, the time average, is between locations $x_{i}\left(t_{0}\right)$ and $x_{i}\left(t_{1}\right)$, the cost of movement from $x_{i}\left(t_{0}\right)$ to $\bar{x}_{i}$ to $x_{i}\left(t_{2}\right)$ is at most the cost of the original movement from $x_{i}\left(t_{0}\right)$ to $x_{i}\left(t_{1}\right)$ to $x_{i}\left(t_{2}\right)$.

Let us now consider the cost of transmission if relay $i$ is placed at location $\bar{x}_{i}$ at time $t_{0}$. Let $d_{i}\left(t_{0}\right)=$ $x_{i+1}\left(t_{0}\right)-x_{i}\left(t_{0}\right)$ and $d_{i}\left(t_{1}\right)=x_{i+1}\left(t_{1}\right)-x_{i}\left(t_{1}\right)$. Define $\bar{d}_{i}$ to be the time average from $t_{0}$ to $t_{2}$ of the transmitting distance between node $i$ and node $i+1$ :

$$
\bar{d}_{i}=\frac{d_{i}\left(t_{0}\right) \cdot\left(t_{1}-t_{0}\right)+d_{i}\left(t_{1}\right) \cdot\left(t_{2}-t_{1}\right)}{t_{2}-t_{0}} .
$$

Observe that

$$
\begin{aligned}
\bar{d}_{i}= & \frac{d_{i}\left(t_{0}\right) \cdot\left(t_{1}-t_{0}\right)+d_{i}\left(t_{1}\right) \cdot\left(t_{2}-t_{1}\right)}{t_{2}-t_{0}} \\
= & \frac{\left(x_{i+1}\left(t_{0}\right)-x_{i}\left(t_{0}\right)\right)\left(t_{1}-t_{0}\right)}{t_{2}-t_{0}} \\
& +\frac{\left(x_{i+1}\left(t_{1}\right)-x_{i}\left(t_{1}\right)\right)\left(t_{2}-t_{1}\right)}{t_{2}-t_{0}} \\
= & \frac{x_{i+1}\left(t_{0}\right) \cdot\left(t_{1}-t_{0}\right)+x_{i+1}\left(t_{1}\right) \cdot\left(t_{2}-t_{1}\right)}{t_{2}-t_{0}} \\
& -\frac{x_{i}\left(t_{0}\right) \cdot\left(t_{1}-t_{0}\right)+x_{i}\left(t_{1}\right) \cdot\left(t_{2}-t_{1}\right)}{t_{2}-t_{0}} \\
= & \bar{x}_{i+1}-\bar{x}_{i}
\end{aligned}
$$

so that it is feasible to place relays $i$ and $i+1$ at their time-averaged locations and obtain the time-averaged transmission distance between them.

By Jensen's inequality,

$$
\left(\bar{d}_{i}\right)^{\alpha} \leq \overline{d_{i}^{\alpha}}=\frac{\left(t_{1}-t_{0}\right) d_{i}\left(t_{0}\right)^{\alpha}+\left(t_{2}-t_{1}\right) d_{i}\left(t_{1}\right)^{\alpha}}{t_{2}-t_{0}}
$$

and thus,

$\left(t_{2}-t_{0}\right)\left(\bar{d}_{i}\right)^{\alpha} \leq \overline{d_{i}^{\alpha}}=\left(t_{1}-t_{0}\right) d_{i}\left(t_{0}\right)^{\alpha}+\left(t_{2}-t_{1}\right) d_{i}\left(t_{1}\right)^{\alpha}$.

That is, the amount of battery depleted for relay $i$ in the time-average case is at most the amount of battery depleted in the case when the relay moves at time $t_{1}$. We thus have the desired result.

Corollary 5. One deployment suffices when maximizing the lifetime of first death.

It also holds that in any optimal solution the transmitter is one of the last to die.

Lemma 6. Given a MAXTL instance, any solution in which the transmitter is not among the last to die is suboptimal.

Proof: Assume the transmitter dies at time $M$, but relay $k$ is the leftmost relay which is still alive in the optimal solution, i.e. relays $1, \ldots, k-1$ die prior to or at time $M$ in the optimal solution. Assume relays $i_{1}, i_{2}, \ldots, i_{l}$ are among the first $k-1$ relays and die at time $M$ with the transmitter.

Move relay $k$ an amount $\phi_{k}$ to the left at time $M-\tau$, where $\tau$ is small enough such that no relay dies in the time interval $[M-\tau, M)$. Since no relay dies, we can assume by Lemma 4 that the relays are stationary in this time interval. Let $\phi_{k}$ be an amount which precludes relay $k$ from dying before time $M$ and is less than $\min \left\{d_{0}(M-\tau), d_{i_{1}}(M-\tau), \ldots, d_{i_{l}}(M-\tau)\right\}$. Sequentially move relays $j=i_{l}, i_{l-1}, \ldots, i_{1}$ at time $M-\tau$, an amount $\phi_{j}$ to the left where $\phi_{j}<\phi_{j+1}$ and is such that

$$
\frac{B_{j}-a \phi_{j}}{\left(d_{j}(M-\tau)-\left(\phi_{j+1}-\phi_{j}\right)\right)^{\alpha}}=\frac{B_{j}}{d_{j}(M-\tau)^{\alpha}} .
$$

Note that such a movement is always feasible: consider the function

$$
J(x)=\frac{B_{j}-a x}{\left(d_{j}(M-\tau)-\left(\phi_{j+1}-x\right)\right)^{\alpha}} .
$$


We have that $J(0)=B_{j} /\left(d_{j}(M-\tau)-\phi_{j+1}\right)^{\alpha}>$ $B_{j} / d_{j}(M-\tau)^{\alpha}$. Furthermore, $J(x)$ is a strictly decreasing function on the interval $\left[0, \phi_{j+1}\right]$ and

$$
J\left(\phi_{j+1}\right)=\frac{B_{j}-a \phi_{j+1}}{d_{j}(M-\tau)^{\alpha}}<\frac{B_{j}}{d_{j}(M-\tau)^{\alpha}} .
$$

Since $J(x)$ is continuous, there is some point $\phi_{j} \in$ $\left[0, \phi_{j+1}\right]$ for which

$$
\frac{B_{j}-a \phi_{j}}{\left(d_{j}(M-\tau)-\left(\phi_{j+1}-\phi_{j}\right)\right)^{\alpha}}=\frac{B_{j}}{d_{j}(M-\tau)^{\alpha}} .
$$

Note that since

$$
\phi_{k}<\min \left\{d_{0}(M-\tau), d_{i_{1}}(M-\tau), \ldots, d_{i_{l}}(M-\tau)\right\}
$$

and $\phi_{k}>\phi_{i_{l}}>\cdots>\phi_{i_{1}}$, relays can never swap order with this shift. This shift of the relays decreases $d_{0}(M-\tau)$, resulting in a solution path with a longer transmission lifetime, a contradiction.

\section{MAXFD On GRID PoInTs}

In this section we consider a discrete version of MAXFD in which relays are deployed on grid points. More specifically, we assume that the final locations $y_{1}, \ldots, y_{n}$ of the relays must be one of the points $j D / m$ for a pre-determined $m$ and $0 \leq j \leq m$. That is, we partition the interval $[0, D]$ into $m$ sub-intervals each of length $\sigma=D / m$ and restrict the final locations from being in the interior of any sub-interval.

Given any $\varepsilon>0$, we show that if $B_{i} \geq a D(1+$ $\left.\frac{1}{n}\right)$, for every $i$, there exists a grid density for which the discrete optimal lifetime of first death is within a factor of $(1+\varepsilon)$ from the optimal lifetime of first death. We provide two algorithms for the discrete problem that are in fact FPTASs for MAXFD, if $B_{i} \geq a D\left(1+\frac{1}{n}\right)$, for every $i$.

We note that our algorithms for the discrete version of MAXFD can be extended to deal with relay deactivations. However, we do not consider deactivations, since they can be ignored if $B_{i} \geq a \max \left\{x_{i}, D-x_{i}\right\}$, for every $i$. (Recall that in this case a relay may effectively deactivate by moving arbitrarily close to the next relay.)

\subsection{Discrete MAXFD vs. MAXFD}

Let $\mathrm{OPT}_{F}$ be the optimal lifetime of first death, and let $\mathrm{OPT}_{F}^{m}$ be the optimal lifetime of first death for the discrete version with $m$ grid points.

We first show that $\mathrm{OPT}_{F}$ and $\mathrm{OPT}_{F}^{m}$ may be far apart even when $m$ is very large. Let $m$ be an odd integer. Consider the following instance with three relays, $a>$ 0 and $D=1$. The relay locations are given by $x=$ $\left(\frac{1}{2}-\frac{\sigma}{2}, \frac{1}{2}+\frac{\sigma}{2}, 1\right)$, and relay batteries are $B_{1}=B_{2}=\frac{a \sigma}{2}$, and $B_{0}=B_{3}=B$, where $B$ is very large. The optimal deployment is $y_{1}=y_{2}=y_{3}=\frac{1}{2}$, and in this case $\mathrm{OPT}_{F}$ is close to $\frac{B}{0.5^{\alpha}}$, while OPT ${ }_{F}^{m}$ is at most $\frac{a}{2 \sigma^{\alpha-1}}$.
Such scenarios may be avoided if the deployment at grid points does not deplete the batteries. In the next lemma we make an assumption that ensures this.

Lemma 7. Let $\varepsilon \in(0,1)$. If $B_{i} \geq a D\left(1+\frac{1}{n}\right)$, for every $i$, and $m=\left\lceil(n+1)^{2} / \varepsilon\right\rceil$, then

$$
\mathrm{OPT}_{F}^{m}>\frac{\mathrm{OPT}_{F}}{(1+\varepsilon)^{\alpha+1}} .
$$

Proof: Let $\left(y_{1}, \ldots, y_{n}\right)$ be an optimal deployment, namely a deployment whose lifetime of first death is $\mathrm{OPT}_{F}$. Also, let $d_{i}=y_{i+1}-y_{i}$, for every $i \in\{0, \ldots, n\}$. Observe that there must exists at least one relay $i$ for which $d_{i} \geq \frac{(n+1) \sigma}{\varepsilon}$, since otherwise

$$
D=\sum_{i=0}^{n} d_{i}<(n+1) \frac{(n+1) \sigma}{\varepsilon}=\frac{(n+1)^{2} D}{\varepsilon m} \leq D .
$$

Let $\ell$ be such a relay.

Let $d_{i}^{\prime}=\sigma \cdot\left\lfloor\frac{d_{i}}{\sigma}\right\rfloor$, for every $i \neq \ell$, and let $d_{\ell}^{\prime}=$ $D-\sum_{i \neq \ell} d_{i}^{\prime}$. Observe that $d_{i}-\sigma<d_{i}^{\prime} \leq d_{i}$, for every $i \neq \ell$, and that

$$
d_{\ell}^{\prime}<d_{\ell}+(n+1) \sigma \leq d_{\ell}+\varepsilon d_{\ell}=(1+\varepsilon) d_{\ell} .
$$

We describe a discrete deployment $y^{\prime}$ using the new distances between relays: $y_{i}^{\prime}=\sum_{k<i} d_{k}^{\prime}$, for every $i$. Observe that

$$
\left|y_{i}^{\prime}-y_{i}\right| \leq n \sigma=\frac{n D}{m} \leq \frac{\varepsilon n D}{(n+1)^{2}}<\frac{\varepsilon D}{n},
$$

for every sensor $i$.

Let $R$ and $R^{\prime}$ are the remaining battery powers after moving to $y$ and $y^{\prime}$, respectively. Since $R_{i} \geq \frac{a D}{n}$, for every $i$, it follows that

$R_{i}^{\prime} \geq R_{i}-a\left|y_{i}^{\prime}-y_{i}\right|>R_{i}-\frac{\varepsilon a D}{n} \geq R_{i}-\varepsilon R_{i}=(1-\varepsilon) R_{i}$,

Putting it all together we get that

$$
L_{i}^{\prime}=\frac{R_{i}^{\prime}}{\left(d_{i}^{\prime}\right)^{\alpha}} \geq \frac{(1-\varepsilon) R_{i}}{(1+\varepsilon)^{\alpha} d_{i}^{\alpha}}>\frac{1}{(1+\varepsilon)^{\alpha+1}} \cdot L_{i},
$$

and we are done.

\subsection{Dynamic Programming Algorithm}

In this section we present a dynamic programming algorithm that solves discrete MAXFD called Lifetime$D P$, shown as Algorithm 1. The running time is polynomial in $n$ and in the number of grid points, $m+1$.

The idea behind this dynamic programming algorithm is to try solving all possible instances for covering the prefix segment $[0, \ldots, j D / m]$ for any $0 \leq j \leq m$ using the $i$ relays closest to the transmitter for $0 \leq i \leq n$.

For $0 \leq j \leq m$ and $0 \leq i \leq n$, let $f(i, j)$ be the lifetime of first death of the solution with the $i$ relays that are closest to the transmitter in which there is a relay with infinite size battery positioned at point $j D / m$ (this is equivalent to moving the receiver to 


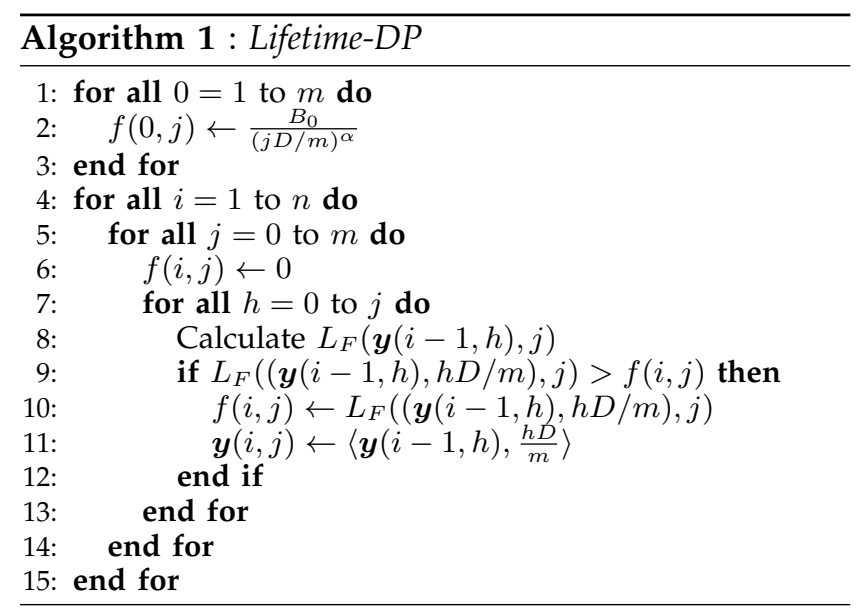

point $j D / m)$. The desired final output will be $f(n, m)$. Initially, for $i=0$ and any $0 \leq j \leq m$, the lifetime is the time it takes the transmitter to die when it transmits to a distance $j D / m$. Also, let $\boldsymbol{y}(i, j)=$ $\left(y_{1}, \ldots, y_{i}\right)$ be the vector of optimal positions assigned to the $i$ relays when transmitting to $j$. Namely, $\boldsymbol{y}(i, j)$ corresponds to $f(i, j)$.

Before showing how to compute $f$ we need the following definition. For $0 \leq j \leq m$, let $L_{F}\left(z_{1}, \ldots, z_{i}, j\right)$ be the lifetime of first death, when there are only $i$ relays, those who are the closest to the transmitter, whose final positions are at points $z_{1}, \ldots, z_{i}$ respectively, that need to cover the prefix segment $[0, \ldots, j D / m]$. The cost of moving relays $1, \ldots, i$ to positions $z_{1}, \ldots, z_{i}$ is taken into account in the solution.

Then, recursively,

$$
f(i, j)= \begin{cases}\max _{0 \leq h \leq j} L_{F}((\boldsymbol{y}(i-1, h), h D / m), j) & i, j>0, \\ \infty & j=0, \\ \frac{B_{0}}{(j D / m)^{\alpha}} & i=0, \\ & j>0 .\end{cases}
$$

That is, look for the lifetime maximizing position for the $i$ th relay among the first $j+1$ possible positions. When a position is examined for the $i$ th relay, use the optimal locations for the first $i-1$ relays transmitting to such a position to find the lifetime for that configuration. Take the maximum lifetime of all position evaluations to be $f(i, j)$.

Lemma 8. Lifetime-DP finds an optimal solution for MAXFD on grid points.

Proof: We proof by induction on $i$ that $f(i, j)$, for every $j$, is the maximum lifetime of first death of the first $i$ relays when the receiver is located at $j D / m$.

Note that the claim is true for $i=0$. Assume the claim is true for all $i-1$. We would like to show that it is true for $i$. In order to compute $f(i, j)$ the algorithm places the $i$ th relay at locations $h=0, \ldots, j D / m$ and considers the lifetime of this placement with the maximal solution of $i-1$ relays transmitting to $h$, $\boldsymbol{y}(i-1, h)$. By the induction hypothesis, $\boldsymbol{y}(i-1, h)$, for $0 \leq h \leq j$, are the positions of maximum lifetime of the first $i-1$ relays transmitting to $h$. Hence, the best lifetime of first death when $i$ is placed at $h D / m$ and transmits to $j$ is the minimum between $f(i-1, h)$ and the lifetime of $i$, namely $L_{F}((\boldsymbol{y}(i-1, h), h D / m), j)$. Since the dynamic programming solution takes the maximum over all $h, f(i, j)$ is optimal for MAXFD on grid points, for every $j$.

The running time of Lifetime-DP is $O\left(n m^{2} \cdot l_{F}(n)\right)$, where $l_{F}(n)$ is the time to compute $L_{F}(N) \cdot l_{F}(n)=$ $O(n)$, since it requires finding the minimum of $n+1$ node lifetimes. Thus, the running time of the dynamic programming algorithm is $O\left(n^{2} m^{2}\right)$.

We note that the running time can be improved to $O\left(n m^{2}\right)$ at the expense of the space complexity using standard techniques.

Because of Lemma 7, Lifetime-DP can be used as an approximation algorithm for MAXFD.

Theorem 9. Lifetime-DP is an FPTAS for the special case of MAXFD in which $B_{i} \geq a D\left(1+\frac{1}{n}\right)$, for every $i$.

\subsection{Binary Search Algorithm}

In this subsection we devise a binary search algorithm called Lifetime Binary Search. We show that this algorithm is an FPTAS for discrete MAXFD. It follows that it is an FPTAS for MAXFD provided that the batteries comply with the conditions of Lemma 7.

Lemma 10. Let $T$ be the optimal lifetime of first death. Given $T^{\prime} \geq 0$, there exists a polynomial-time algorithm that determines whether $T^{\prime} \leq T$.

Proof: Initially set $y_{n+1}=D$. Going from $i=n$ to 1 we try to move relay $i$ to the leftmost position for which relay $i$ has lifetime $T^{\prime}$. If we succeed for all $i$, we check whether the transmitter has enough power to transmit to relay 1 at least until $T^{\prime}$.

If $x_{i}<y_{i+1}$, move relay $i$ to the leftmost position $y_{i}$ in the closed interval $\left[x_{i-1}, y_{i+1}\right]$, for which relay $i$ has lifetime $L_{i} \geq T^{\prime}$, if such a location exists. Finding $y_{i}$ can be done by solving the following equations:

$$
\frac{B_{i}-a p_{i}}{\left(d_{i}+p_{i}\right)^{\alpha}}=T^{\prime},
$$

if $L_{i} \geq T^{\prime}$, and

$$
\frac{B_{i}+a p_{i}}{\left(d_{i}+p_{i}\right)^{\alpha}}=T^{\prime},
$$

if $L_{i}<T^{\prime}$, where $p_{i}=x_{i}-y_{i}$. Note that $y_{i}$ exists if $L_{i} \geq T^{\prime}$. In addition it exists if $L_{i}<T^{\prime}$ and $B_{i}-a d_{i}>$ 0 . If $y_{i}$ exists, update $d_{i-1} \leftarrow d_{i-1}+\left(y_{i}-x_{i}\right)$ and $d_{i} \leftarrow d_{i}+\left(x_{i}-y_{i}\right)$. Otherwise output NO.

If $x_{i}=y_{i+1}=\cdots=y_{i+k}$, for some $k \geq 1$, proceed as follows. Move relays $i, \ldots, i+k$ to the left as long as $i, \ldots, i+k-1$ have battery power and $i+k$ has lifetime at least $T^{\prime}$ and until reaching $x_{i-1}$. Computing the maximum distance $i+k$ may travel can be done 
as explained in the case of $x_{i}<y_{i+1}$. The maximum distance relay $i+j$, for $j<k$, may travel is $B_{i+j}^{\prime} / a$ where $B_{i+j}^{\prime}$ is the remaining battery power of relay $i+j$. If all relays have enough battery power to be moved to $x_{i-1}$ with relay $i+k$ still having lifetime at least $T^{\prime}$, update $y_{i+j} \leftarrow x_{i-1}$, for $j \in\{0, \ldots, k\}$ and move to iteration $i-1$. Otherwise, the move to the left was stopped at $x \leq x_{i}$ by relay $j$ : either $j<k$ and the battery of relay $i+j$ was depleted, or $j=k$ and the lifetime of relay $i+k$ is exactly lifetime $T^{\prime}$. Assign $y_{i+\ell} \leftarrow x$, for every $\ell \in\{j, \ldots, k\}$, and continue the process of moving $i$ with $k=j-1$.

There are $n+1$ relays, each may cause $O(n)$ movements. In each such movement we check $O(n)$ relays and solve either (6) or (7).

In the discrete MAXFD case, instead of solving the equations, we find the leftmost grid point that can be reached using the battery. This is $O(m)$ time in the worst case. Equation (7) is monotone decreasing and hence the requested grid point can be found in $O(\log m)$ time using binary search.

Let $T_{a}$ denote the lifetime for movement cost $a$. Lifetime Binary Search performs a binary search for $T$ in the interval $\left[T_{\infty}, T_{0}\right]$.

Theorem 11. Lifetime Binary Search is an FPTAS for discrete MAXFD.

Proof: Observe that $T_{a} \geq T_{b}$ if $a \leq b$. It follows that $T_{a} \in\left[T_{\infty}, T_{0}\right]$. By Theorem 2 we have that

$$
T_{0}=\frac{\left(\sum_{j=0}^{n} \sqrt[\alpha]{B_{j}}\right)^{\alpha}}{D^{\alpha}} \leq \frac{\left(n \sqrt[\alpha]{B_{\max }}\right)^{\alpha}}{D^{\alpha}}=\frac{n^{\alpha} B_{\max }}{D^{\alpha}},
$$

where $B_{\max }=\max _{i} B_{i}$. On the other hand,

$$
T_{\infty}=\min _{i} \frac{B_{i}}{\left(x_{i+1}-x_{i}\right)^{\alpha}} \geq \frac{B_{\min }}{D^{\alpha}},
$$

where $B_{\min }=\min _{i} B_{i}$. Hence the number of iterations of the binary search is bounded by

$\log \left(\frac{T_{0}}{\varepsilon T_{\infty}}\right) \leq \log \left(\frac{n^{\alpha} B_{\max }}{\varepsilon B_{\min }}\right)=\alpha \log n+\log \frac{B_{\max }}{B_{\min }}+\log \frac{1}{\varepsilon}$,

which is polynomial in the input size and $1 / \varepsilon$.

Due to Lemma 7 Lifetime Binary Search can be used to approximate MAXFD.

Theorem 12. Lifetime Binary Search is an FPTAS for the special case of MAXFD where $B_{i} \geq a D\left(1+\frac{1}{n}\right)$, for every $i$.

\section{HeURISTICS for MAXTL}

In this section we develop heuristics for single deployment MAXTL, when $a>0$. The reason for considering single deployment MAXTL is due to experimental evidence that suggests that any optimal MAXTL solution requires just one deployment, see section 7.2.

\subsection{Lifetime-DP and NLP as Heuristics for MAXTL}

If instead of evaluating the function $L_{F}(N)$, Lifetime$D P$ evaluates $L_{T}(N)$ in each iteration, it can be used as a heuristic for single deployment MAXTL. $L_{T}(N)$ can be computed by summing over successive computations of the lifetime of first death until the death of the transmitter. Since the lifetime of first death on $k$ nodes can be computed in time $O(k), L_{T}(N)$ for $n+1$ nodes can be computed in worst-case time $O\left(n^{2}\right)$. The running time of this dynamic programming heuristic which we call DP is $O\left(n m^{2} \cdot l_{T}(n)\right)$, where $l_{T}(n)$ is the time to compute $L_{T}(N)$. Since $l_{T}(n)$ is $O\left(n^{2}\right)$, the running time of $D P$ is $O\left(n^{3} m^{2}\right)$.

Single deployment MAXTL can also be formulated as a nonlinear program if $L_{T}(N)$ is used as the objective function in (2). We implement a nonlinear programming algorithm NLPalgo for this formulation using the MATLAB function FMINCON which we set to use the medium-scale line-search method. This method finds local maxima and is not guaranteed to find the global maximum.

\subsection{Other Algorithms and Heuristics for MAXTL}

We now describe the MtoOpt algorithm as shown in Algorithm 2. Since we know the set of optimal locations when there is no friction, $O p t_{a=0}$, we would like to move all the relays a fixed percentage towards these optimal locations under the assumption that the optimal location for a relay in the presence of friction is somewhere between the relay's initial location and $O p t_{a=0}$. The algorithm evaluates each of the solutions where the relays move $1 \%, 2 \% \ldots, 100 \%$ towards $O p t_{a=0}$ and outputs the solution resulting in the longest transmission lifetime. This algorithm runs in $O\left(n^{2}\right)$ time as $l_{T}(n)$ is of order $O\left(n^{2}\right)$ and a constant number of points are evaluated.

We also implement an exponential algorithm, EXP. This algorithm does not use any heuristic but instead uses brute force to evaluate all possible $\left(\begin{array}{c}m+1 \\ n\end{array}\right)$ combinations for the $n$ relays over the $m+1$ possible, equally spaced, grid locations between the transmitter and the receiver. The algorithm preserves order among the relays. The EXP runs in $O\left(\mathrm{~m}^{n}\right)$ time and, hence, is only practical for small values of $n$ when over 100 grid points are considered.

Algorithm 2 The MtoOpt algorithm

\section{INPUT: $N$}

Calculate the set of optimal locations, Opt $t_{\text {stat }}$, using Eqn. 5 For Each Percentage $i$ do:

1) $L_{i} \longleftarrow$ Lifetime $\left(\right.$ InitLoc $+\left(O p t_{\text {stat }}-\right.$ InitLoc $\left.) * i\right)$

2) If $L(i)>$ BestLifetime then

a) BestLifetime $\longleftarrow L(i)$

b) BestLocs « InitLoc $+\left(O p t_{\text {stat }}-\right.$ InitLoc $\left.) * i\right)$

OUTPUT: Final locations of the relays. 


\section{Simulations and Results}

This section summarizes the results of the comprehensive simulation study that was performed. The simulations were performed using MATLAB 6.5 R13 on a machine running Windows XP 2002 SP3 with an Intel Pentium D CPU 2.8GHz and 2GB RAM. For a fixed unit length network with one transmitter and one receiver we varied each of the parameters $n, \alpha$, $a$ and $m$, where $n$ is the number of nodes in the network, $\alpha$ is the path loss exponent in the communication model, $a$ is the coefficient of friction and $m$ is the number of discrete points that are considered as possible final locations in single deployment MAXTL with grid relay locations. For each range of values of these parameters we evaluated their effect by running simulations with all the mobile relays being initially located at the transmitter, at the midway point between the transmitter and the receiver, and at the receiver.

We also simulated the relays being randomly distributed along the line according to both the uniform distribution and the normal distribution. In the random initial location cases we used Monte Carlo simulations and present the results with $95 \%$ confidence intervals.

We evaluate each of the heuristic algorithms in the following ways: $i$ ) how they perform against the upper bound lifetime achievable when there is no friction and relays can be located at any point, $i i$ ) how they perform against the lower bound given by the case where mobility has infinite cost and the nodes must remain in their initial locations, and iii) the time cost of each of the algorithms. In general, i) illustrates the cost of mobility and the effect of increasing the number of relays in a network while ii) demonstrates the benefit that mobile nodes bring to a WSN and how, even with relatively high friction, they can extend the lifetime of a network.

We define a unit of friction to be that which allows a relay with one unit of battery power to travel the unit distance from the transmitter to the relay. In other words, with friction set to 1 a relay with full battery will use up all of its power moving from the transmitter to the receiver.

\subsection{Performance of Algorithms for MAXFD}

The performance of all the algorithms and heuristics from the preceding two sections was tested on the MAXFD problem. The effects of varying the initial locations of the relays, their initial battery levels and the cost of movement were simulated. Also, for the discrete algorithms, the effect of varying the number of grid points available was tested. On the MAXFD problem, the Lifetime Binary Search, the Lifetime-DP and the EXP algorithms had very similar performances with over 50 gridpoints although they were sensitive to the exact number of points used. Above
200 gridpoints, the algorithms performed nearly identically to the NLPalgo-FD.

\subsection{Super Exponential Algorithm}

We conducted extensive simulations to decide whether allowing the relays to move more than once during the lifetime of the network results in an increase of network lifetime in the MAXTL problem. To this end, we created a super-exponential algorithm that pre-computes the entire lifetime of the network for each possible configuration of the $n$ relays on the $m$ possible locations. For each of the $\left(\begin{array}{c}m+1 \\ n\end{array}\right)$ configurations the SuperEXP algorithm with the fitness function Lifetime of First Death was re-run after the first node died on the $\left(\begin{array}{c}m+1 \\ n-1\end{array}\right)$ remaining reconfigurations. This was repeated for the $n-2, n-3, \ldots 1$ relays or until the transmitter died. Also, we evaluated exponential versions of the DP and MtoOpt algorithms where we reconfigure the network after each relay death and use Lifetime of First Death to evaluate the optimal configuration at each iteration. However, as the SuperEXP outperforms both DPexp and MtoOpt-exp for large $m$, we will only present our results from simulations using this algorithm.

Our simulations show that while the SuperEXP often outperforms the EXP and DP algorithms for levels of friction close to 0 , the EXP and $D P$ are just as good as SuperEXP for all other levels of friction. Furthermore, the super EXP algorithm never outperforms the NLPalgo. Our empirical observations lead us to the following conjecture:

Conjecture 13. In an optimal MAXTL solution, the relays need only be deployed once.

Based on this conjecture, in the remaining simulations we evaluate the algorithms on a move once basis with the remaining relays adjusting their transmission ranges after the death of a relay to accommodate for the gap in communication.

Another important observation from analyzing the exponential versions of the heuristic algorithms is that they perform just as well when the battery levels are not equal across the nodes in the network. After the death of the first relay, the remaining nodes will have already moved different amounts and communicated over different distances and so, when the heuristics are applied to the reduced set of nodes in the second iteration the battery levels across the nodes in this sub-problem will be unequal. Therefore, in evaluating the performance of our algorithms we set the initial battery levels to be one unit across all nodes in the simulations that follow.

\subsection{How many grid points do we need?}

By varying the value of $m$ between 5 and 200 in steps of 5 , while holding all the other parameters constant, we evaluated the effect that increasing the 
number of possible final locations for the relays in the EXP and DP algorithms had on the optimal locations found by these algorithms. We observed that the optimal lifetimes found by the heuristic algorithms increased asymptotically towards the upper bound with $m$. Increasing $m$ from 5 to 65 results in an increase of the worst lifetime found by the algorithms by $28 \%$ in absolute terms, while increasing $m$ from 100 to 200 results in an increase of just $1 \%$. However, the EXP algorithm takes 340.422 seconds to run when $m=200$ and only 41.437 seconds when $m=100$. The $D P$, by contrast, takes 3.859 and 15.578 seconds when $m=100$ and $m=200$, respectively. Since using large values of $m$ was impractical for our simulations, especially those requiring the monte carlo method, we set $m=100$ when evaluating the EXP algorithm against other parameters except where explicitly stated otherwise. The Lifetime Binary Search algorithm was extremely fast and could easily accommodate high values of $m$ but was highly inconsistent at values $<100$.

Note that the EXP algorithm outperforms the DP for many values of $m$, this is due to the algorithms being evaluated using Transmission Lifetime as opposed to the Lifetime of First Death.

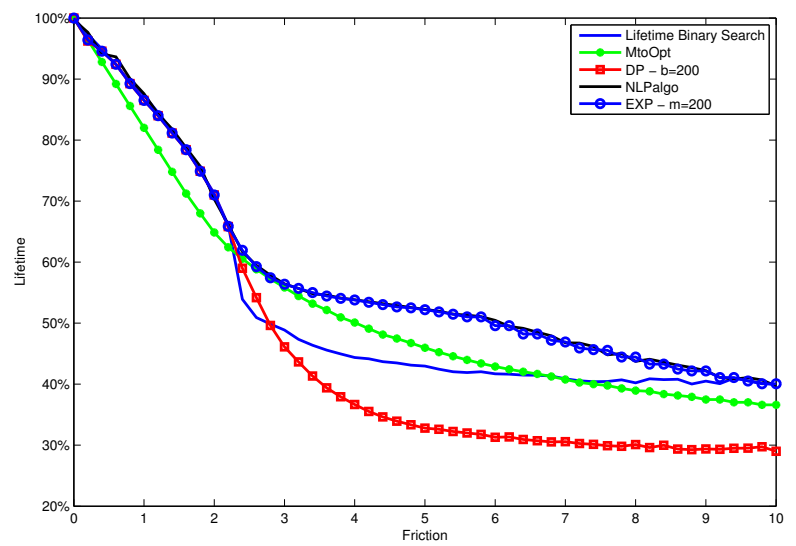

Fig. 2: Performance of the heuristics compared to the optimal non-friction solution

\subsection{The Effect of $\alpha$ on Network Lifetime}

Using the values $1 \leq \alpha \leq 6, B_{i}=1, n=4$ and varying the cost of mobility between 0 and 10 we investigated the effect path loss exponent has on the performance of the algorithms and the resulting optimal lifetimes. We found that the parameter $\alpha$ has little effect on the respective algorithms for values greater than 1 other than to consistently reduce the optimal lifetimes found. Consequently, we chose to set $\alpha=2$ in the simulations to follow.

\subsection{Increasing the Relay Count: How do the algo- rithms scale?}

Using the values $2 \leq n \leq 30, B_{i}=1, \alpha=2$ and varying the cost of mobility between 0 and 10 we evaluated the effect the number of relays has on the algorithms. We found that increasing the number of mobile relays in the network has the expected effect of increasing the lifetime of the network. However, the increase is consistent across the algorithms and, hence, the number of relays in the network did not affect the performance of our algorithms. For the below analysis, we fix the number of relays at $n=4$ so that we can compare results across all experiments including those requiring monte carlo simulations.

One important observation from our simulations is that the NLPalgo does not scale well: for values of $n$ over 40 the DP algorithm with $m=200$ finds more optimal solutions when $a>0.8$.

Note, we were unable to test the EXP and SuperEXP algorithms for values of $n>11$ with $m>100$ as $M A T L A B$ was unable to fully compute the possible permutations.

\subsection{The Effect of Friction}

Using the values $\alpha=2, B_{i}=1, n=4, m=200$ and setting the cost of mobility, $a$, between 0 and 10 we get the graph in Fig. 2. Out of all the algorithms we evaluated, the NLPalgo algorithm gave the longest MAXTL lifetimes across the entire range of parameter settings. The dynamic programming approach, $D P$, performs as well as the EXP for levels of friction below 2.5 but its performance quickly drops off above this level. MtoOpt gives surprisingly good results considering its simplicity and for $a \geq 2.5$ it outperforms the DP. The Lifetime Binary Search also performs optimally at low levels of friction but also drops off above $a=2.5$. Note that it outperforms the $D P$ at higher levels of friction which is surprising considering that it is designed for the MAXFD problem.

All of the algorithms we evaluated passed the sense checks that for zero friction the relays should go to the locations described by Eqn. 5 and for infinite friction the relays should remain in their initial locations. More importantly, all of the algorithms performed at least as well as the case where the relays must remain in their initial locations and from this we can conclude that it always pays to move unless friction is effectively infinite or the relays are initially located at their optimal positions.

\subsection{Random Locations}

To fully understand the effect of the initial locations of the relays on the maximum lifetime achievable by a network we ran monte carlo simulations on network lifetime using each of the heuristic algorithms with the initial locations of the relays randomly chosen from 
the uniform and normal distributions while varying the level of friction and keeping all other parameters constant. We implemented the Control Variate technique (using the theoretical optimal locations) and Antithetic Variate technique to reduce the standard deviation in the mean value of the simulations by a factor of 3 .

We present the results from the uniform distribution simulations with 50,000 iterations of the NLPalgo algorithm and $95 \%$ confidence interval error bars in Fig. 3. Clearly, we can see that increasing the friction has the combined effect of decreasing the average lifetime in the networks and increasing the standard deviation. This means that the initial locations of the relays has a greater impact on the achievable lifetime of a network as the level of friction is increased. Also, this impact grows rapidly: increasing the level of friction from 0 to 2 results in an increase in standard deviation from 0 to $4.96 \%$ resulting in error bars of $\pm 9.72 \%$ around the mean.

\subsection{Time cost of algorithms}

The heuristic algorithms were fast relative to the exponential algorithms and especially the SuperEXP algorithm. For $a=10, m=200, B_{i}=1, n=4$ and $\alpha=2$ the Lifetime Binary Search algorithm ran the fastest by far at $0.0023 \mathrm{~s}$, the MtoOpt algorithm was next fastest at $0.047 \mathrm{~s}$, the NLPalgo and DP algorithms were comparable at $16.56 \mathrm{~s}$ and $15.94 \mathrm{~s}$, respectively. The exponential algorithms on the same set of parameters all took between $475 \mathrm{~s}$ and $500 \mathrm{~s}$.

To give an idea of how slow the SuperEXP is, on the same set of parameters as before but with $n=3$ the SuperEXP took $481.9 \mathrm{~s}$ while the EXP took $5.7 \mathrm{~s}$.

The monte carlo simulations were only run on the Lifetime Binary Search, MtoOpt, NLPalgo and DP algorithms with $n \leq 4$ and $a \leq 2$ with the simulations taking up to 7 hours for the larger parameter values.

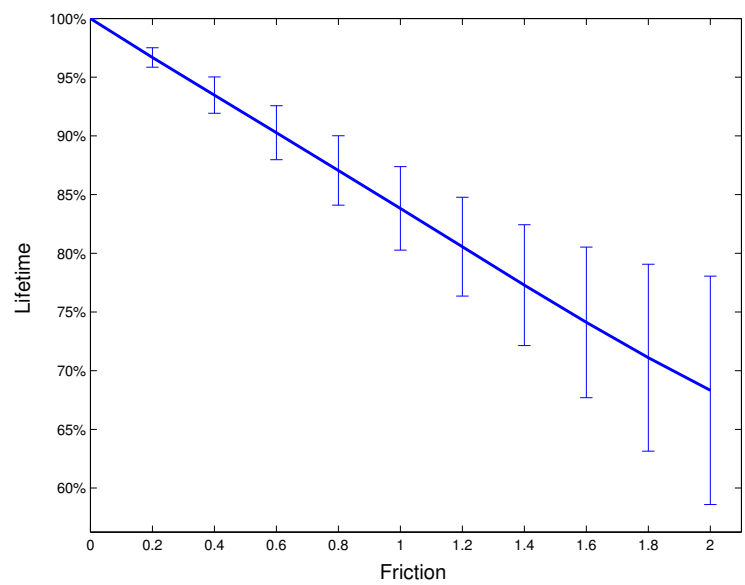

Fig. 3: MC simulations of lifetimes for random starting locations with 95\% Error Bars

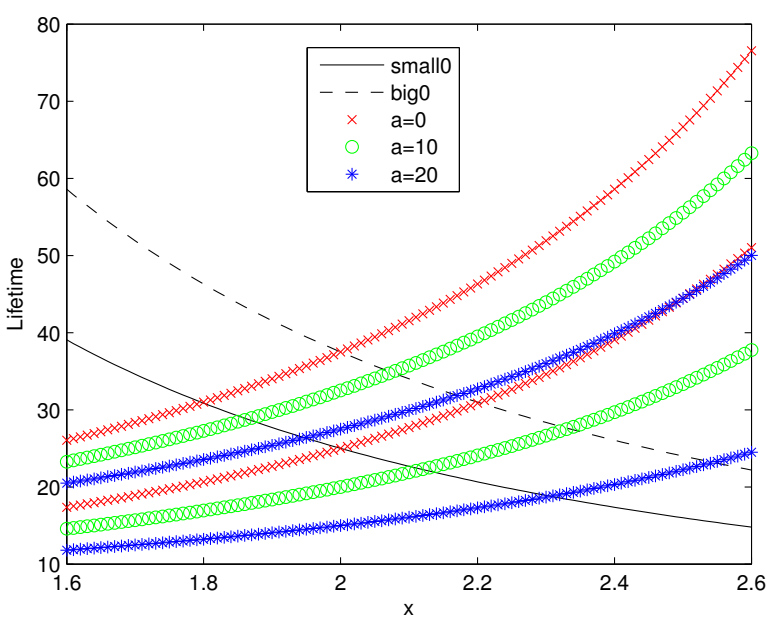

Fig. 4: Instance of simulation in experimental verification of Conjecture 14

\subsection{Deployment from Base Station}

Assume $n$ relays with battery powers $B_{1}, \ldots, B_{n}$ are initially positioned with the transmitter at 0 . In what order should the relays be deployed to maximize the lifetime of first death? We can verify experimentally that relays with larger batteries should be deployed to positions farther than relays with smaller batteries.

Conjecture 14. If all relays have initial locations at 0 , it is optimal for them to be ordered after deployment in increasing order of initial battery power.

Assume that in an optimal solution there are two adjacent relays $s$ and $t$ with initial battery powers $B_{s}<B_{t}$ but $y_{s}>y_{t}$ after deployment. Note that if relays are not ordered in increasing order of initial battery power there is always such a pair. Assume relay $m$, located at $y_{m}$ is the relay which relay $s$ transmits to after deployment.

Let $y=y_{t}$. Consider the interval $I=\left(y, y_{m}\right]$. Let $B_{s}^{\prime}$ and $B_{t}^{\prime}$ denote the battery levels of relays $s$ and $t$ after traveling from 0 to location $y$. It must be that $B_{s}^{\prime}<B_{t}^{\prime}$. Consider case 1 where relay $t$ remains at $y$ and relay $s$ moves to location $y+x$ in interval $I$. For this case the lifetime of relay $t$ is $B_{t}^{\prime} / x^{\alpha}$ and the lifetime of relay $s$ is $\left(B_{s}^{\prime}-a x\right) /\left(y_{m}-x\right)^{\alpha}$. Consider case 2 where relay $s$ remains at $y$ and relay $t$ moves to location $y+x$ in interval $I$. For this case the lifetime of relay $s$ is $B_{s}^{\prime} / x^{\alpha}$ and the lifetime of relay $t$ is $\left(B_{t}^{\prime}-a x\right) /\left(y_{m}-x\right)^{\alpha}$.

Assume $a$ is large enough so that $\left(B_{s}^{\prime}-a x\right) /\left(y_{m}-\right.$ $x)^{\alpha}$ does not intersect with $B_{t}^{\prime} / x^{\alpha}$ in case 1 . Since $\left(B_{t}^{\prime}-a x\right) /\left(y_{m}-x\right)^{\alpha}>\left(B_{s}^{\prime}-a x\right) /\left(y_{m}-x\right)^{\alpha}$, and by assumption $\left(B_{s}^{\prime}-a x\right) /\left(y_{m}-x\right)^{\alpha}<B_{t}^{\prime} / x^{\alpha} \forall x \in I$, the lifetime of first death of case 2 is greater than the lifetime of first death of case 1, contradicting the initial assumption of optimality.

For the $a$ for which $\left(B_{s}^{\prime}-a x\right) /\left(y_{m}-x\right)^{\alpha}$ intersects with $B_{t}^{\prime} / x^{\alpha}$ (intersection point is maximum lifetime 
of first death), we verify that lifetime of first death of case 2 is greater than the lifetime of first death of case 1 via simulation. An instance of the simulation is shown in Fig 4.

In Fig. $4, B_{t}^{\prime}=150, B_{s}^{\prime}=100, \alpha=2$ and $I=$ $(0,4]$. Big0 is the function $150 / x^{2}$ and smallo is the function $100 / x^{2}$. There are also the functions which depend on $a: f_{150}(a, x)=(150-a x) /(4-x)^{\alpha}$ and $f_{100}(a, x)=(100-a x) /(4-x)^{\alpha}$. Keep in mind that $(150-a x) /(4-x)^{\alpha}>(100-a x) /(4-x)^{\alpha}$ for a given $a$ and $\forall x \in I$ when viewing Fig. 4 . That is, for a given $a, f_{150}(a, x)$ will be the larger function.

When $a=0, f_{150}(0, x)$ and $f_{100}(0, x)$ intersect smallo and big0 respectively at exactly the same lifetime. Note that when $a=10, f_{150}(10, x)$ intersects small0 at a larger lifetime than does $f_{100}(10, x)$ intersect big0. When $a=20$, the difference in lifetimes of the intersection points is even more drastic. This behavior is common across all experiments. Thus, there is strong experimental evidence supporting the conjecture.

\section{Future RESEARCH}

First, we list several open problems:

- Formally proving conjectures 13 and 14

- We conjecture that MAXTL is NP-Hard when there is friction.

Finally, there are a number of possible natural generalizations for our problem:

- Consider the model which allows swapping and/or activation/deactivation.

- Consider the model where the initial and final locations of the relays can be anywhere in the plane.

\section{References}

[1] R. Appuswamy, E. Atsan, C. Fragouli, and M. Franceschetti. On relay placement for deterministic line networks. In Wireless Network Coding Conference (WiNC), 2010 IEEE, pages 1-9, June 2010.

[2] Stefano Basagni, Alessio Carosi, Emanuel Melachrinoudis, Chiara Petrioli, and Z. Maria Wang. Controlled sink mobility for prolonging wireless sensor networks lifetime. Wireless Networks, 14(6):831-858, 2008.

[3] Arnab Chakrabarti, Ashutosh Sabharwal, and Behnaam Aazhang. Using predictable observer mobility for power efficient design of sensor networks. In Feng Zhao and Leonidas J. Guibas, editors, IPSN, volume 2634 of Lecture Notes in Computer Science, pages 129-145. Springer, 2003.

[4] Anurag Chandra, Deepak Bansal, Rajeev Shorey, Ashutosh Kulshreshtha, and Manish Gupta. Characterization of mobility patterns based on cell topography in a cellular radio system. In IEEE ICPWC, pages 428-432, 1999.

[5] M. Di Francesco, S.K. Das, and G. Anastasi. Data collection in wireless sensor networks with mobile elements: A survey. In Transactions on Sensor Networks (TOSN), 2012 ACM To Appear, February 2012.

[6] Fatmé El-Moukaddem, Eric Torng, and Guoliang Xing. Maximizing data gathering capacity of wireless sensor networks using mobile relays. In MASS, pages 312-321. IEEE, 2010.

[7] David Kiyoshi Goldenberg, Jie Lin, and A. Stephen Morse. Towards mobility as a network control primitive. In Jun Murai, Charles E. Perkins, and Leandros Tassiulas, editors, MobiHoc, pages 163-174. ACM, 2004.
[8] Sushant Jain, Rahul Shah, Waylon Brunette, Gaetano Borriello, and Sumit Roy. Exploiting mobility for energy efficient data collection in wireless sensor networks. MONET, 11(3):327-339, 2006.

[9] Zhen Jiang, Jie $\mathrm{Wu}$, and Robert Kline. Mobility control for achieving optimal configuration in wireless sensor networks. Wireless Networks, 16(1):141-152, 2010.

[10] Philo Juang, Hidekazu Oki, Yong Wang, Margaret Martonosi, Li-Shiuan Peh, and Daniel Rubenstein. Energy-efficient computing for wildlife tracking: design tradeoffs and early experiences with zebranet. In ASPLOS, pages 96-107, 2002.

[11] Aman Kansal, Mohammad Rahimi, William J Kaiser, Mani Srivastava, Gregory J Pottie, and Deborah Estrin. Controlled mobility for sustainable wireless networks. In IEEE SECON, 2004.

[12] Hyung Seok Kim, Tarek F. Abdelzaher, and Wook Hyun Kwon. Minimum-energy asynchronous dissemination to mobile sinks in wireless sensor networks. In Ian F. Akyildiz, Deborah Estrin, David E. Culler, and Mani B. Srivastava, editors, SenSys, pages 193-204. ACM, 2003.

[13] Vivek Mhatre, Catherine Rosenberg, Daniel Kofman, Ravi Mazumdar, and Ness B. Shroff. A minimum cost heterogeneous sensor network with a lifetime constraint. IEEE Trans. Mob. Comput., 4(1):4-15, 2005.

[14] Thomas Moscibroda, Roger Wattenhofer, and Aaron Zollinger. Topology control meets sinr: : the scheduling complexity of arbitrary topologies. In Sergio Palazzo, Marco Conti, and Raghupathy Sivakumar, editors, MobiHoc, pages 310-321. ACM, 2006.

[15] Rahul C. Shah, Sumit Roy, Sushant Jain, and Waylon Brunette. Data mules: modeling and analysis of a three-tier architecture for sparse sensor networks. Ad Hoc Networks, 1(2-3):215-233, 2003.

[16] Guangming Song, Yaoxin Zhou, Zhigang Wei, and Aiguo Song. A smart node architecture for adding mobility to wireless sensor networks. Sensors and Actuators A: Physical, 147(1):216 - 221, 2008.

[17] Guiling Wang, Guohong Cao, and Thomas F. La Porta. Movement-assisted sensor deployment. IEEE Trans. Mob. Comput., 5(6):640-652, 2006.

[18] Guiling Wang, Mary Jane Irwin, Piotr Berman, Haoying Fu, and Thomas F. La Porta. Optimizing sensor movement planning for energy efficiency. In Kaushik Roy and Vivek Tiwari, editors, ISLPED, pages 215-220. ACM, 2005. 